\title{
Exascale Computer System
}

National Cancer Institute

\section{Source}

National Cancer Institute. Exascale Computer System. NCI Thesaurus. Code C139306.

Refers to computer systems capable of a quintillion (10^18) calculations each second.

This computing power is expected to facilitate the analysis of data sets that would require too much time with currently available systems. Such analysis would allow more realistic simulations of the complex processes and relationships behind many natural processes. This will have practical applications in everything from precision medicine to regional climate, water use to materials science, nuclear physics to national security. 